\title{
Apoyo público e innovación a nivel de firmas
}

\author{
Andrés Jung ${ }^{1 *}$, Diego Karsaclian ${ }^{1}$
}

Resumen: Este artículo pretende aportar a la literatura que analiza los efectos de las políticas públicas sobre la innovación a nivel de firmas, a partir del caso de una pequeña economía en desarrollo, Uruguay. Analiza la relación entre apoyo público y probabilidad de innovar para el mercado, e introduce un rezago entre el apoyo otorgado y la innovación realizada, de manera de valorar rezago en el impacto. El análisis econométrico utiliza Probit binomiales con corrección de Heckman, para corregir sesgos de selección. Se concluye que los apoyos públicos importan a los efectos de innovar para el mercado, ya sea porque estimulan la innovación o porque la facilitan en empresas que ya innovan. Asimismo, las actividades de innovación de empresas asociadas a apoyos públicos, en Uruguay, no parecen requerir de largo tiempo de maduración. Finalmente, se sugiere profundizar el estudio de la relación entre internacionalización de las empresas y probabilidad de innovar.

Palabras claves: innovación; subsidios; evaluación de política.

Abstract: Title: Public support and innovation at the firm level. This article intends to contribute to the analyses of the effects of public policies on innovation at the firm level, based on the case of a small developing economy (Uruguay). It studies the relation between public support and the probability of innovating at the market level, introducing a lag between support and innovation. The econometric analysis uses binomial Probit models with Heckman sampling bias correction. The article concludes that public support matters for innovation at the market level, whether by stimulating innovation or by facilitating it in already innovative firms. Moreover, the innovation activities of Uruguayan firms receiving public support do not seem to require a long time to develop. Finally, it is suggested that the relation between firm internationalization and the probability of innovating be further studied.

Keywords: innovation; subsidies; policy evaluation.

Submitted: March $10^{\text {th }} 2017 /$ Approved: September $25^{\text {th }} 2017$

\section{Introducción}

El desarrollo económico se asocia actualmente a procesos de diversificación productiva que, a través de la generación de capacidades por parte de los agentes económicos, conducen a economías más complejas y con mayor potencial de crecimiento (Hausmann \& Klinger, 2006; Hausmann \& Rodrik, 2003; Imbs \& Warcziarg, 2003).

Para diversificar la matriz productiva y transformar las economías, se requiere que las empresas innoven. En países en desarrollo, las innovaciones que tienen estos efectos no deben ser necesariamente algo nuevo para el mundo. Aunque su alcance sea el mercado (Hausmann \& Rodrik, 2003), pueden tener un impacto significativo, asociado con el desempeño a nivel de firma y con los aumentos en la productividad total de los factores a nivel nacional (Navarro et al., 2016; Maloney y Perry, 2005). Este hecho es particularmente relevante para los países de América Latina, donde las deficiencias en la capacidades nacionales de innovación han sido una de las principales barreras para el crecimiento (Maloney, 2002).

Esto explica que, de manera creciente, los países presten atención a los procesos de innovación en las empresas y desarrollen políticas para promoverlos, y que a nivel académico se analice la relación entre políticas de innovación y desempeño innovador. Esta relación ha sido ampliamente estudiada para países desarrollados, aunque no lo ha sido tanto para países emergentes.
Las políticas de apoyo a la innovación abarcan un amplio espectro de objetivos y acciones. Si bien la innovación se materializa cuando se transforman nuevas ideas en soluciones sociales y económicas (Navarro et al.., 2016), principalmente por parte de empresas públicas o privadas (Edquist, 2011), o el conocimiento en valor económico (Camacho et al., 2010), la propia inversión en actividades de innovación (ej. en I+D) ya es un componente crítico para el crecimiento a largo plazo (Navarro et al.., 2016). Estas actividades no solo generan nuevo conocimiento asociado a una innovación, sino que son importantes para desarrollar capacidad de absorción, es decir para adquirir y adaptar tecnologías existentes a nuevas situaciones (Cohen \& Levinthal, 1989; García-Granero et al., 2014).

El hecho de que las políticas se orienten tanto a facilitar el acceso al mercado de una nueva idea ya concebida, como a promover conocimiento que permita generar nuevos productos o procesos, que luego serán llevados al mercado, pone de manifiesto la relevancia de considerar los tiempos requeridos para que una medida de apoyo a la innovación logre tener el impacto deseado. Este es un aspecto escasamente analizado en la literatura. Puede esperarse que apoyos orientados a generar conocimiento (ej. I+D), requieran de mayor tiempo para manifestar sus efectos que apoyos más cercanos a la introducción de un nuevo producto o proceso en el mercado.

Este tema es relevante para países en desarrollo. Como plantean Szczygielski et al. (2017), las políticas públicas de apoyo a la inno-

(1) Departamento de Economía, Universidad Católica del Uruguay, Montevideo, Uruguay

*Autor de correspondencia: ajung@ucu.edu.uy 
vación en las empresas en países 'seguidores en tecnología' pueden ayudar o no a las economías a avanzar en la 'escala tecnológica'. Pero no todas las políticas son pertinentes para ello y, en particular, es importante considerar el rezago entre apoyo público e innovación a nivel de firma.

El objetivo de este artículo es aportar a la creciente literatura que analiza los efectos de las políticas públicas sobre la innovación a nivel de firmas, a partir del caso de una pequeña economía en desarrollo, como es Uruguay. Los aportes principales son dos: a) analiza la relación entre el otorgamiento de apoyos públicos y la probabilidad de las firmas de innovar a nivel de mercado, ya que es a este nivel que las innovaciones aportan a la transformación de las economías; y b) introduce en el análisis un rezago entre el apoyo otorgado y la innovación realizada por las empresas, de manera de valorar los tiempos de impacto y, en consecuencia, reflexionar sobre el tipo de apoyo otorgado. Ambos aspectos son importantes, ya que si las innovaciones a nivel de mercado son las que transforman las economías (Cowling, 2016), es posible focalizar mejor las políticas y, en función de la 'cercanía al mercado' de los instrumentos utilizados, puede valorarse la rapidez de su impacto esperado en términos de innovación.

El artículo se estructura de manera que, luego de esta introducción, se discuten los aspectos más relevantes de la literatura (sección 2), y en la sección 3 se presentan los datos y la metodología de análisis. En la cuarta sección se discuten los principales resultados del análisis empírico, para finalmente concluir en la sección 5.

\section{Las políticas públicas y la innovación}

Los argumentos conceptuales en que se basan las políticas de innovación provienen básicamente de dos corrientes teóricas: la neoclásica y la evolucionista.

Desde la primera, la principal justificación de la intervención pública se basa en la idea de que los niveles de inversión en innovación por parte de las empresas tienden a ser menores a lo socialmente óptimo, debido a la existencia de fallas de mercado. En general, éstas se asocian al uso de poder de mercado, la existencia de externalidades y las asimetrías de información.

La principal falla de mercado que identifica la literatura es la existencia de externalidades positivas. La innovación involucra la generación de conocimiento cuya completa protección no es posible o es costosa y, a pesar de que la imitación tiene sus costos (Hall \& Lerner, 2009), termina comportándose como un bien público. Como consecuencia, las empresas innovadoras no pueden incorporar todos los beneficios de sus inversiones en innovación, lo que determina una brecha entre el óptimo social y el privado (Aboal \& Garda, 2015).

El enfoque evolucionista, por su parte, está vinculado a los sistemas nacionales de innovación. En este caso, la intervención pública se justifica para mitigar eventuales fallas que tienen que ver con la conectividad y las capacidades individuales y organizacionales para el funcionamiento de un sistema de innovación (Edler et al., 2013). Estos sistemas están constituidos por un conjunto de agentes económicos, instituciones y prácticas que participan de manera relevante en los procesos de innovación. Desde un enfoque complementario, también pueden definirse a partir de actividades o determinantes de la innovación (Edquist, 2011). Los actores de estos sistemas a nivel nacional son, entre otros, empresas, universidades, agencias públicas, sistemas financieros y mercados, que contribuyen a generar, diseminar, usar, adaptar e incorporar conocimiento en los sistemas de producción y en la sociedad (Freeman, 1987; Metcalfe, 1995), conformando el marco en el que se desarrollan las políticas de apoyo a la innovación por parte de los gobiernos (Navarro et al., 2016).

Las políticas públicas se justifican de manera creciente en una orientación proactiva para acelerar y viabilizar ciertos desarrollos que se consideran socialmente deseables (Edler et al., 2013). Muchas veces no se explicitan las lógicas sobre las que se basa la formulación de las políticas y los análisis se centran en los impactos que estas pueden alcanzar y no en los problemas que podrían resolver (Edquist, 2011). La evaluación de los impactos, sin embargo, es compleja. Hay varias razones por las que las políticas de apoyo a la innovación podrían no tener los efectos buscados (Andrews \& Criscuolo, 2013). Además del conocido "crowding out" del apoyo público sobre la inversión privada en innovación, pueden generarse incentivos para la captura de rentas por parte de las empresas, lo que puede llevar a una mala asignación de los recursos disponibles por parte de las agencias responsables de las políticas.

Por otra parte, las políticas de innovación deben articularse con otras, orientadas a promover la competitividad empresarial y a mejorar el complejo entorno en el que las empresas desarrollan su actividad (ej. Durán et al., 2016; Padilla-Pérez \& Gaudin, 2013; Edquist, 2011; Magro \& Wilson, 2013; Dogson et al., 2011). Las condiciones de contexto y las capacidades de las empresas, condicionan el efecto de los instrumentos directos de apoyo a la innovación (Edler et al., 2013), por lo cual resulta importante prever acciones de mejora en diversos niveles para un mayor impacto (Cowling, 2016; Andrews \& Criscuolo, 2013). La interacción entre las políticas también puede generar efectos no previstos, ya que nuevos instrumentos son introducidos en contextos donde ya existen otras políticas (Cunningham et al., 2013, Flanagan et al., 2011, OCDE, 2010). Finalmente, el horizonte temporal necesario para evaluar los resultados de la política de innovación son extensos y, al menos en América Latina, los instrumentos son modificados antes de que se pueda concluir una evaluación real de su impacto (Durán et al., 2016).

Existe una amplia literatura que analiza empíricamente la relación entre políticas de apoyo a la innovación y el comportamiento innovador de las empresas. La mayor parte se refiere a países desarrollados y, en particular, al sector manufacturero. Esta literatura se ha centrado en su mayoría en los efectos de las políticas sobre la magnitud del gasto en I+D realizado por las empresas (ej. Afcha, 2012) y en la evaluación de la existencia o no de "crowding-out" de los fondos públicos sobre la inversión privada. En cuanto al efecto sobre la inversión en I+D, en general se verifica una relación positiva con el apoyo público a nivel de firmas. En relación al "crowding-out", David et al. (2000) repasaron 35 años de evidencia econométrica acumulada al respecto y encontra- 
ron efectos de sustitución solo en un tercio de los estudios.

En cuanto a la literatura que analiza el efecto de las políticas sobre el desempeño innovador de las empresas, los resultados no son del todo concluyentes. Así, Aerts y Czarnitzki (2004) analizaron los efectos de las políticas de innovación sobre las solicitudes de patentes para empresas flamencas, sin encontrar resultados significativos. Czarnitzki et al. (2011), en cambio, sí encontraron un impacto positivo de las políticas en el número de innovaciones en producto introducidas por empresas canadienses del sector manufacturero, con efectos incluso en innovaciones para el mercado (tanto local como internacional), al igual que Czarnitzki y Hussinger (2004) para el caso de un grupo de empresas alemanas. Cowling (2016) estudió los efectos de créditos impositivos orientados a promover la innovación en el caso de PYMES británicas. Encontró evidencia de que la herramienta fomentó las innovaciones en procesos de las empresas, y que este efecto se potenciaba cuando la empresa contaba con altos niveles de planificación estratégica y de capacidad.

Edler et al. (2013) realizaron un compendio de la evidencia disponible, revisando más de 800 informes de evaluación de efectividad de políticas de innovación implementadas en países desarrollados. Encontraron que el conjunto de la evidencia sugiere que las medidas fiscales tienen un efecto positivo en las innovaciones incrementales (en oposición a las radicales) y en las innovaciones en procesos, aunque no se ha comprobado que estas tengan efectos en la productividad de las empresas. En particular, la evidencia acerca de los efectos de las medidas de subsidio directo en los resultados innovadores de las empresas es contradictoria.

En el caso de los países emergentes, donde los problemas de información son mayores, los resultados tampoco son concluyentes. Szczygielski et al. (2017), analizan los casos de Polonia y Turquía como países 'seguidores en tecnología' respecto a países avanzados, encontrando que el apoyo a I+D se asocia positivamente con la innovación a nivel de firma, aunque no así el subsidio para la generación de capacidades. Para América Latina, Hall y Maffioli (2008) compendiaron los resultados de evaluaciones que realizó el BID sobre los efectos de políticas de fondos de desarrollo tecnológico para Argentina, Brasil, Chile y Panamá entre 2005 y 2007. Por un lado, la evidencia que analizaron sugiere que los fondos no generan efecto sustitución y que estos afectan positivamente la intensidad del gasto en $\mathrm{I}+\mathrm{D}$, además de favorecer una actitud más proactiva respecto a las actividades de innovación. De cualquier manera, no encontraron resultados estadísticamente significativos de impacto en el número de patentes, ventas de productos innovadores, o crecimiento en la productividad de las empresas, probablemente debido al escaso horizonte de tiempo en el que las evaluaciones fueron realizadas. Aboal y Garda (2015) analizaron el caso de Uruguay, concluyendo que el apoyo público incrementó el gasto privado en innovación, el gasto privado en $\mathrm{I}+\mathrm{D}$ y el número de ventas innovadoras en las empresas. Sin embargo, sus resultados muestran que el otorgamiento de apoyos no habría tenido efectos considerables sobre el gasto privado en innovación por parte de empresas que invertirían en este tipo de actividades incluso si no recibiesen apoyo público.
Si bien la evidencia empírica no es concluyente para países en desarrollo, en términos de los efectos de las políticas públicas sobre el comportamiento innovador de las empresas, existen indicios de una asociación positiva (ej. Cowling, 2016; Czarnitski et al., 2011; Edler et al., 2013). En particular, ello parece ser el caso para países de América Latina (Hall \& Maffioli, 2008), lo que hace relevante el tema ya que es una región que tiene deficiencias en sus sistemas nacionales de innovación (Maloney, 2002). Dado que, como señala Cowling (2016), las innovaciones 'para el mercado' son las que promueven la transformación de las economías hacia estructuras más diversificadas, complejas y con mayor potencial de crecimiento, lo que es importante para el desarrollo de los países (Hausmann et al., 2007; Hausmann \& Rodrik, 2003), resulta de interés evaluar la posible asociación positiva entre el apoyo público y la probabilidad de introducir productos o procesos que sean nuevos para el mercado (sea local o internacional).

A partir de lo anterior, se fomula la siguiente hipótesis:

Hipótesis 1: Existe una asociación positiva entre el otorgamiento de apoyo público a las empresas para desarrollar actividades de innovación y la probabilidad de que estas innoven a nivel de mercado (local e internacional).

En todo caso, el análisis de la relación entre los apoyos públicos y el comportamiento innovador de las empresas debe incorporar información referida a varios períodos. Una de las dificultades para evaluar el efecto de las políticas hace referencia al tiempo requerido para que los instrumentos tengan efectos sobre los resultados de la actividad innovadora de la empresa (Edler et al. 2013). Este es un aspecto relevante ya que apoyos más orientados a la generación de conocimiento (ej. I+D) pueden requerir un tiempo mayor de maduración. El uso de instrumentos orientados a apoyar la transformación de conocimiento en valor de mercado, puede mostrar resultados más rápidamente. Incluso, se han identificado casos en los cuales un efecto positivo de ciertos tipos de apoyo público en el comportamiento innovador de las empresas en el corto plazo, se diluye en el tiempo (ej. Bakhshi et al.. 2013). Szczygielski et al. (2017), incorporan este aspecto en su análisis evaluando la asociación entre apoyos realizados en un período y actividades innovadoras en un período siguiente. Ello parece interesante en el caso de países de América Latina, en los que la inversión en conocimiento (ej. I+D) es relativamente baja, y existen numerosos instrumentos orientados a apoyar la transformación de conocimiento en valor (Durán et al., 2016), lo que permitiría esperar una mayor cercanía en el tiempo entre apoyo recibido e innovación realizada.

En función de lo anterior, para el caso de estudio, se formula la siguiente hipótesis:

Hipótesis 2: La probabilidad de que una empresa innove a nivel de mercado (local e internacional) es mayor cuando recibe apoyo público en períodos cercanos a la introducción de la innovación en el mercado. 


\section{Datos y metodología}

\section{Datos}

Para el análisis se fusionaron las bases de las rondas 2007-2009 y 2010-2012 de la Encuesta de Actividades de Innovación a empresas uruguayas que lleva a cabo la ANII (Agencia Nacional de Investigación e Innovación).

La muestra quedó conformada por 1459 empresas, 716 pertenecientes al sector manufacturero y 743 al sector de servicios, con personal ocupado promedio mayor o igual a 5 personas y con ventas mayores o iguales a 120 millones de pesos. La encuesta cubre la totalidad de los subsectores manufactureros y parte de los subsectores de servicios ${ }^{1}$. Al fusionar las bases, se dejaron de lado aquellas empresas con menos de 4 años de actividad a 2012, y se eliminaron algunos casos que podían distorsionar el análisis (básicamente empresas públicas y microempresas).

A los efectos del estudio, se consideraron innovaciones en producto y en proceso, incorporando en esta última categoría innovaciones en organización y comercialización. Como puede esperarse dada su complementariedad (Cowling, 2016), en ambos sectores se detecta una fuerte correlación entre innovación en producto y en procesos: $76 \%$ de las empresas industriales y $74 \%$ de las de servicios que innovaron en procesos también innovaron en producto. Asimismo, dada su importancia en términos de transformación de la estructura productiva, se consideraron las innovaciones 'para el mercado, sea éste local o internacional (ej. Cowling, 2016; Szczygielski et al., 2017), distinguiéndolas de las innovaciones 'para la empresa'.

En la tabla 1 se presentan el número de empresas innovadoras para ambos sectores, así como la cantidad de empresas por tipo de innovación (como porcentaje del total de empresas de cada sector) y la cantidad de empresas por alcance de sus innovaciones (como porcentaje del total de empresas innovadoras en el sector respectivo).

Tabla 1. Características de la muestra

\begin{tabular}{l|r|r}
\hline & \multicolumn{1}{|c}{ Industria } & \multicolumn{1}{c}{ Servicios } \\
\hline Número de observaciones & 716 & 743 \\
\hline Empresas innovadoras & 278 & 238 \\
Porcentaje del total & $38,8 \%$ & $32,0 \%$ \\
\hline Tipo de innovación (como porcentaje de las empresas innovadoras) \\
\hline Producto & $52,9 \%$ & $48,7 \%$ \\
\hline Proceso & $87,1 \%$ & $87,4 \%$ \\
\hline Alcance máximo de la innovación (como porcentaje de las empresas \\
innovadoras) \\
\hline Empresa \\
\hline Mercado
\end{tabular}

Fuente: Elaboración propia en base a ANII

* Incluye innovaciones en organización y comercialización

${ }^{* *}$ Incluye innovaciones a nivel internacional
En la tabla 2 se presentan las medias de distintas variables para las empresas no innovadoras, innovadoras en general e innovadoras para el mercado de cada sector. Se observa que las empresas innovadoras tienen una mayor cantidad de años en actividad, mayor cantidad de personal total y de personal calificado, mayor intensidad exportadora y mayor porcentaje de empresas con capital extranjero. La productividad laboral de las empresas innovadoras es mayor en la industria pero no en los servicios. En general estos rasgos se profundizan a nivel de empresas que innovan para el mercado, excepto para el caso de la productividad de la mano de obra.

Las empresas innovadoras que utilizan las herramientas de apoyo público son una minoría y son un grupo relativamente menor en el sector servicios (13.5\% de las empresas innovadoras) que en el industrial (34.5\% de las empresas innovadoras). El proceso de selección permite acceder a los apoyos a la mayor parte de las empresas que lo solicitan: el $86.5 \%$ de las empresas industriales y un $74.4 \%$ de las de servicios que solicitan apoyo lo obtienen.

La fuente de apoyo a la que recurren mayoritariamente la empresas (sean industriales o de servicios) para desarrollar actividades de innovación es la Ley de inversiones (72\% de las empresas industriales y $59 \%$ de las de servicios), si bien no es un instrumento diseñado específicamente para la promoción de la innovación. Esta Ley prevé un crédito fiscal por inversiones, que se aprueba caso a caso, y que mejora en general las condiciones de competitividad de las empresas. Estas inversiones pueden incluir incorporación de nuevos equipamiento y tecnologías y otras inversiones asociadas al proceso de innovación. La segunda fuente en importancia es el apoyo canalizado a través de la ANII, que consiste en subsidios directos a actividades de innovación que también son aprobadas caso a caso (18\% de las empresas industriales y $27 \%$ de las de servicios recibieron apoyo de esta fuente). Existen otras fuentes de menor peso relativo. En todo caso, la muestra permite determinar si las empresas que accedieron a estos apoyos públicos lo recibieron en el período 2007-2009 (un 29\%), en el periodo 2010-2012 (54\%), o en ambos (17\%).

(1) Incluye empresas pertenecientes a las secciones: D. Suministro de electricidad, gas, vapor y aire acondicionado, E. Suministro de agua; alcantarillado, gestión de desechos y actividades de saneamiento (excluyendo las Divisiones 37 y 39), H. Transporte y almacenamiento, I. Alojamiento y servicio de comida, J. Información y comunicación, M. Actividades profesionales, científicas y técnicas, N. Actividades administrativas y servicios de apoyo, y Q. Servicios sociales y relacionados con la Salud Humana (excluyendo las Divisiones 87 y 88). Los subsectores considerados en la encuesta representaban en $201063 \%$ del VAB y $58 \%$ del personal ocupado en el sector 
Tabla 2. Estadísticas descriptivas

\begin{tabular}{|c|c|c|c|c|c|c|}
\hline \multirow{2}{*}{$\begin{array}{l}\text { Sector } \\
\text { Resultados innovadores }\end{array}$} & \multicolumn{3}{|c|}{ Industria } & \multicolumn{3}{|c|}{ Servicios } \\
\hline & $\begin{array}{l}\text { No } \\
\text { innova }\end{array}$ & Innova & $\begin{array}{l}\text { Innova para el } \\
\text { mercado }\end{array}$ & No innova & Innova & $\begin{array}{l}\text { Innova para el } \\
\text { mercado }\end{array}$ \\
\hline Edad (años) & 29,0 & 33,7 & 33,7 & 22,1 & 25,5 & 25,5 \\
\hline Intensidad exportadora & $14,7 \%$ & $21,0 \%$ & $23,0 \%$ & $5,8 \%$ & $7,1 \%$ & $7,9 \%$ \\
\hline $\begin{array}{l}\text { Capital extranjero (porcentaje que } \\
\text { tiene capital extranjero). }\end{array}$ & $10,3 \%$ & $19,4 \%$ & $18,4 \%$ & $10,9 \%$ & $17,2 \%$ & $26,7 \%$ \\
\hline
\end{tabular}

Fuente: Elaboración propia en base a ANII

\section{Metodología}

El propósito del análisis es estimar en qué medida la probabilidad de que una empresa innove a nivel de mercado está asociada al hecho de que haya recibido o no apoyo público para financiar actividades de innovación y al momento en que lo recibió. Para el análisis econométrico se utilizaron modelos Probit binomiales con método de corrección de Heckman (Heckprobit) para mitigar potenciales problemas de sesgos de selección en las muestras (ej. Cowling, 2016; David et al., 2000; Szczygielski et al., 2017).

El problema de sesgo de selección surge del hecho de que solo es posible observar el nivel de alcance de las innovaciones de las empresas que efectivamente tuvieron alguna innovación. El método de Heckman, que se desarrolla en dos etapas, consiste en estimar un primer modelo "de selección" que estima la probabilidad que tiene una empresa de innovar (cualquiera sea el nivel de alcance) para luego incorporar las estimaciones transformadas de este primer modelo como variables explicativas adicionales al modelo "principal", que estima las probabilidades de que la innovación haya logrado ser novedosa a nivel de mercado. Específicamente, para controlar la posibilidad de selectividad implícita al excluir a aquellas empresas que no son innovadoras, las estimaciones del modelo principal son corregidas por un "regresor de selección", estimado utilizando el modelo de selección. La variable dependiente de este último modelo toma el valor 1 si la empresa tuvo algún tipo de innovación y 0 si no lo tuvo.

El modelo de selección fue especificado con un mayor número de variables explicativas que el modelo principal. Las variables incorporadas fueron dummies subsectoriales y de localización geográfica (Montevideo - resto del país). Siguiendo a Cowling (2016), estas variables fueron introducidas pues podrían captar efectos de derrame dentro de sistemas de innovación sectoriales o regionales que aumentan la probabilidad de innovar.
Formalmente, los modelos Probit simples estiman la probabilidad de que la empresa innove a nivel de mercado $\operatorname{Pr}\left(Y_{i}=1 \mid X_{i}\right)$ como una función de sus variables independientes. La estimación se realiza a través del método de máxima verosimilitud. De esta forma se tiene que:

$$
\operatorname{Pr}\left(Y=1 \mid X_{i}\right)=\Phi\left(X_{i} \beta+\varepsilon\right)
$$

Donde Xi es un vector de variables independientes, $\beta$ sus coeficientes y la $\Phi$ función de distribución acumulativa de la distribución normal estándar, que transforma cualquier valor real en un número (probabilidad) entre 0 y 1.

El modelo Probit con sesgo de selección supone que existe una relación subyacente entre la variable dependiente y las independientes, representada a través de la ecuación latente:

$$
Y_{j}^{*}=X_{j} \beta+\varepsilon_{1}
$$

Tal que solo es observable el resultado binario:

$$
Y_{j}^{\text {probit }}=\left(y_{j}^{*}>0\right)
$$

A fin de captar los efectos de las variables no observadas (en este caso las que no innovan), se estima mediante un Probit simple una ecuación de selección que asume el valor 1 si se cumple:

$$
Z_{\mathrm{j}} \gamma+\varepsilon_{2}>0
$$

Donde el vector de variables independientes Zj equivale al Xi más las variables adicionales subsectoriales y de localización geográfica. Las estimaciones de este modelo son incorporadas al modelo principal. Por último, se tiene que:

$$
\operatorname{corr}\left(\varepsilon_{1}, \varepsilon_{2}\right)=\rho
$$


Donde $\rho$ es la correlación entre los errores de las dos ecuaciones estimadas (la del modelo de selección y la del modelo principal). Cuando $\rho \neq 0$, existe una correlación entre los términos de error de ambas ecuaciones, por lo que un modelo Probit simple produciría resultados sesgados. El método Heckprobit corrige este sesgo y provee estimadores consistentes y eficientes para los parámetros del modelo.

De esta manera, se estima para cada sector (industria y servicios) y cada tipo de innovación (producto o proceso) un modelo cuya variable dependiente es la probabilidad de que la empresa introduzca una innovación a nivel de mercado. En todos los casos, el modelo de selección tiene por variable dependiente si la empresa tuvo una innovación del tipo correspondiente al del modelo principal (esto es, producto - producto, proceso - proceso) cualquiera haya sido su alcance. En todos los casos, las variables dummies de innovación corresponden al segundo período de los datos de la encuesta (2010-2012). En el caso de las innovaciones en producto para el sector servicios, se estimó un modelo Probit simple, dado que no se encontró evidencia de sesgo de selección.

Se realizaron diversas pruebas adicionales a los efectos de comprobar la robustez de los resultados. Se estimaron los modelos considerando únicamente a las empresas que no recibieron apoyo público para la innovación en el segundo período y se realizaron las pruebas de hetorescedasticidad de Breusch-Pagan / Cook-Weisberg, rechazándose la hipótesis nula de varianza constante en todos los casos. En función de estos resultados, todos los modelos fueron estimados con errores estándares robustos.

\section{Variables independientes}

La tabla 3 contiene detalles sobre la operacionalización de las variables explicativas a introducir en los modelos, la que sigue criterios habitualmente utilizados en la literatura (ej. Cowling, 2016; Czarntiski \& Lopes-Bento, 2011).

Tabla 3. Operacionalización de variables

\begin{tabular}{l|l}
\hline Variable & Definición \\
\hline Apoyo & $\begin{array}{l}\text { La empresa recibió apoyo del Estado para financiar actividades innovadoras. En el modelo la variable identifica cuatro } \\
\text { situaciones distintas: si recibió apoyo en el primer período, si recibió apoyo en el segundo, si recibió apoyo en ambos, o si } \\
\text { no recibió apoyo. Las fuentes de apoyo estatal son: ANII, CARPE, Ley de Inversiones (COMAP), Fondo Industrial (DNI) } \\
\text { y Otras instituciones. }\end{array}$ \\
\hline Edad & Cantidad de años transcurridos desde que la empresa inició actividades hasta el último año de la encuesta (2012). \\
\hline Tamaño & $\begin{array}{l}\text { Cantidad de personal empleado, promedio anual del período 2010 - 2012. } \\
\text { Porcentaje del ingreso por venta proveniente de ventas al exterior, promedio anual del período 2010 - 2012. Se introduce } \\
\text { en el modelo como cuatro dummies: No exportadora (0\%), 0\%>25\%, 25\%>80\% y 80\%>100\%. }\end{array}$ \\
\hline Capital extranjero & $\begin{array}{l}\text { Variable dummy, adopta el valor } 1 \text { si al menos parte del capital de la empresa es de origen extranjero y 0 si no tiene capital } \\
\text { extranjero. }\end{array}$ \\
\hline Empleo calificado & $\begin{array}{l}\text { Porcentaje del personal que cuenta con algún tipo de calificación (cantidad de técnicos y profesionales con los que contaba } \\
\text { la empresa en el año } 2012 \text { sobre el total de empleados en 2012). }\end{array}$ \\
\hline Productividad laboral & $\begin{array}{l}\text { Promedio de ventas anuales en el período } 2010 \text { - 2012 sobre promedio de personal ocupado en ese mismo período (en } \\
\text { pesos corrientes) }\end{array}$ \\
\hline
\end{tabular}

Las variables de edad, tamaño, empleo calificado y productividad laboral fueron introducidas en el modelo con una transformación logarítmica debido a la fuerte distribución asimétrica hacia la derecha que presentan sus datos.
En la tabla 4 se presentan las matrices de correlación entre las variables explicativas del modelo para ambos sectores. Ninguna de las correlaciones representa un problema para la estimación. 
Tabla 4. Matrices de correlaciones

\begin{tabular}{|c|c|c|c|c|c|c|}
\hline Industria & Apoyo & Edad & Tamaño & Intensidad Exp. & Capital Ext. & Empleo calificado \\
\hline Edad & $0.1426^{*}$ & & & & & \\
\hline Tamaño & $0.2347^{\star}$ & $0.2369^{*}$ & & & & \\
\hline Intensidad exp. & $0.0777^{\star}$ & 0.0206 & $0.3824^{*}$ & & & \\
\hline Capital ext. & 0.0385 & 0.0290 & $0.3807^{*}$ & $0.3901^{*}$ & & \\
\hline Empleo calificado & -0.0290 & -0.0177 & $-0.4701^{\star}$ & -0.0424 & 0.0625 & \\
\hline Prod. laboral & $0.1471^{\star}$ & $0.2008^{*}$ & $0.3373^{*}$ & $0.3542^{*}$ & $0.3352^{*}$ & 0.0644 \\
\hline Servicios & Apoyo & Edad & Tamaño & Intensidad Exp. & Capital Ext. & Empleo calificado \\
\hline Edad & 0.0553 & & & & & \\
\hline Tamaño & $0.1792^{*}$ & $0.2314^{*}$ & & & & \\
\hline Intensidad exp. & 0.0507 & -0.0651 & -0.0431 & & & \\
\hline Capital ext. & 0.0222 & -0.0668 & $0.1423^{*}$ & $0.2073^{*}$ & & \\
\hline Empleo calificado & 0.0434 & -0.0223 & $-0.2181^{\star}$ & 0.0282 & 0.0328 & \\
\hline Prod. laboral & $0.0726^{*}$ & $0.1790^{*}$ & -0.0726 & $0.2338^{*}$ & $0.2520^{*}$ & $0.1861^{\star}$ \\
\hline
\end{tabular}

\section{Resultados}

El análisis de los resultados (Tabla 5) muestra, para todos los casos analizados, que efectivamente existe una asociación positiva y significativa entre el apoyo público y la probabilidad de introducir innovaciones a nivel de mercado por parte de las empresas. Los coeficientes positivos indican que las empresas que han recibido apoyo público tienen mayor probabilidad de innovar para el mercado (sea local o internacional), en comparación con una empresa que no lo ha recibido. Si bien no es posible comprobar el sentido de la causalidad, ello permite confirmar la Hipótesis 1, independientemente del sector (industria o servicios) y del tipo de innovación (producto o proceso).

Tabla 5. Modelos estimados

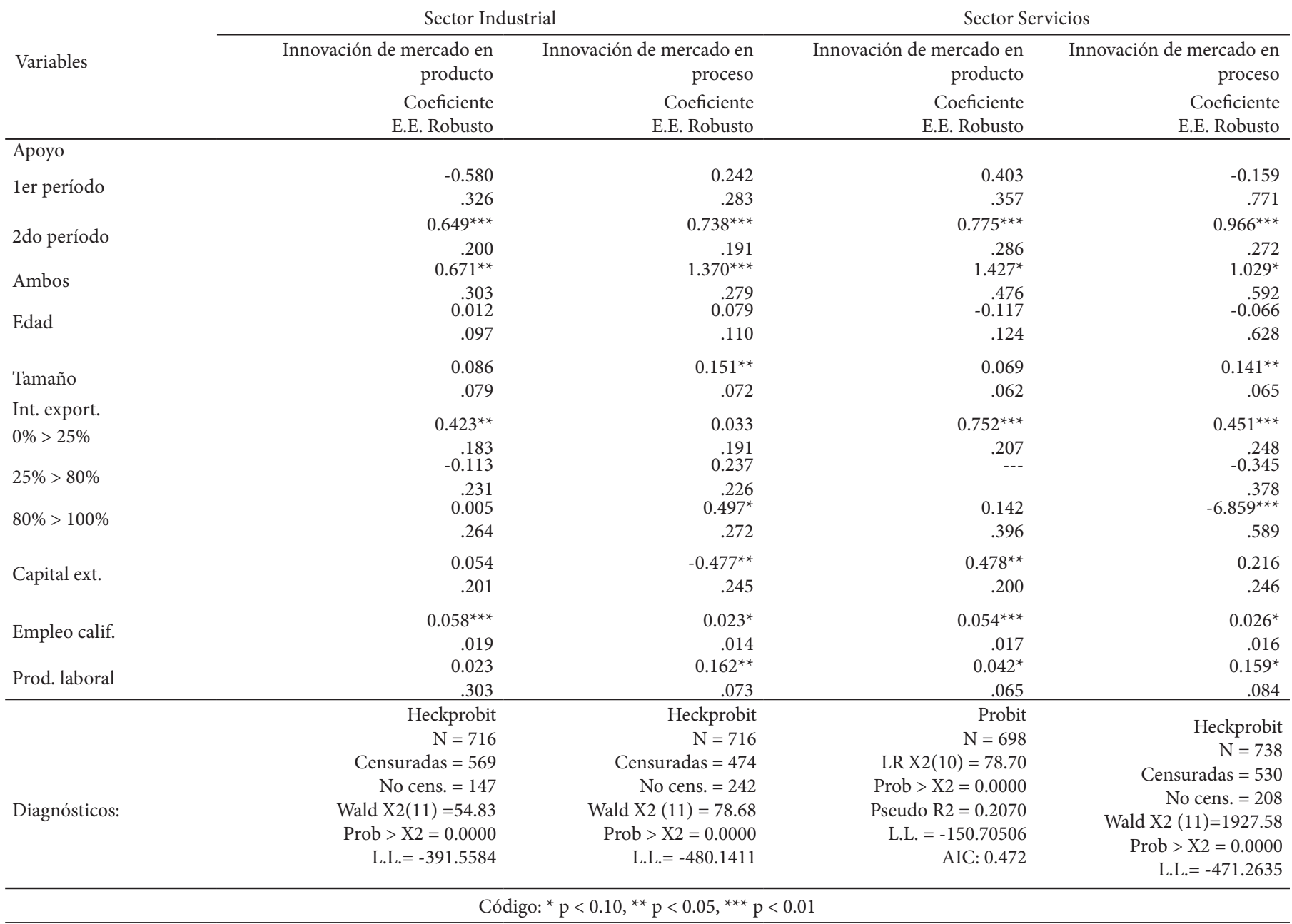

ISSN: 0718-2724. (http://jotmi.org) 
En segundo término, se observa que la asociación es positiva y significativa siempre que se haya recibido un apoyo en el mismo período (de 3 años) en el que la empresa innovó (2010-2012), incluso en el caso en que este apoyo se venga dando desde un período anterior (20072009). No se encontraron asociaciones significativas entre los apoyos otorgados solamente en el período anterior al que corresponde a la innovación y la probabilidad de innovar para el mercado. Este resultado permite validar la Hipótesis 2. La cercanía temporal entre apoyo e innovación para el mercado sugiere que las empresas solicitan (o reciben) apoyo sólo una vez que han decidido o están procesando la introducción de la innovación.

En cuanto a las variables de control, en el caso de innovaciones en producto para ambos sectores y en procesos para los servicios, se observa una asociación positiva entre la probabilidad de innovar nivel de mercado y ser exportador con una intensidad exportadora de hasta $25 \%$ de las ventas. Para el sector industrial, en el caso de innovaciones en procesos, esta asociación se verifica para una alta intensidad exportadora, lo que podría explicarse por la estructura de exportaciones de Uruguay y la necesidad de sus empresas de ser más eficientes para competir en los mercados internacionales, más que de introducir nuevos productos en dichos mercados. Para los servicios, en el caso de innovación en procesos, se observa una asociación negativa y significativa con la intensidad exportadora de más de $80 \%$. Los resultados estarían indicando que las empresas de servicios no exportadoras o con una relativamente baja intensidad exportadora tienen mayores probabilidades de innovar a nivel de mercado en procesos que las empresas de alta intensidad exportadora.

En cuanto al tamaño, el porcentaje de personal calificado y la productividad laboral, los resultados van en línea con lo esperado. La presencia de capital extranjero no muestra resultados significativos, salvo una asociación positiva en el caso de innovación en producto en los servicios.

\section{Conclusiones}

La preocupación de los países por generar procesos de desarrollo de sus economías a través de mayor productividad, diversificación y complejidad de su estructura productiva, ha puesto de relieve la importancia de las políticas de apoyo a la innovación a nivel de las empresas. En particular, el mayor énfasis está puesto en la introducción de productos y procesos nuevos 'para el mercado' (sea local o internacional), dado su impacto en la transformación de la estructura productiva (Cowling, 2016).

El tema adquiere mayor relevancia considerando el contexto de la innovación en América Latina y, en particular, en Uruguay. Respecto a los estándares de la OCDE, estos países exhiben niveles bajos de inversión y de resultados en innovación, así como de calidad educativa y de recursos humanos destinados a la investigación (Maloney \& Perry, 2005). Este contexto debilita a los sistemas nacionales de innovación y pone de relieve la importancia de las políticas de apoyo. Lo que no está claro es la efectividad de las políticas aplicadas. Este artículo pretende realizar un pequeño aporte en este sentido, desde el caso de Uruguay. Los resultados obtenidos permiten extraer algunas conclusiones que, si bien con limitaciones, pueden aportar sugerencias para el futuro.

En primer lugar, y aunque sin poder identificar causalidades, se concluye que los apoyos públicos importan para la innovación a nivel de mercado, ya sea porque estimulan a las empresas a innovar o porque facilitan esta actividad en las empresas que ya lo hacen o tienen decidido hacerlo. Dado que la amplia mayoría de las empresas que recibieron apoyo lo hicieron a través de la Ley de Inversiones, instrumento que no tiene un foco específico en promover la actividad innovadora (Durán et al., 2016; Aboal et al., 2015), parece relevante repensar la estructura de apoyos públicos con un sesgo más "proinnovación".

En segundo término, se observa que las actividades de innovación que desarrollan las empresas asociadas a los apoyos públicos, en el caso de Uruguay, no requieren largo tiempo de maduración. Probablemente más que actividades de generación de conocimiento se trate de actividades de incorporación y adaptación de productos y tecnologías vigentes en otros países. Esto destaca la importancia del fortalecimiento de la capacidad de absorción de las empresas lo que les permitirá sacar partido de vínculos y generación de conocimiento externo. Esto que ha sido comprobado para el caso del emprendimiento innovador en países en desarrollo (Gonzalez-Pernía et al., 2015), parece también aplicable a este caso.

En tercer lugar, si bien no es el foco del análisis, se observa que en tres de los cuatro casos estudiados existe una relación positiva entre algún grado de internacionalización de las empresas a través de su intensidad exportadora y la probabilidad de innovar. Existe una extensa literatura sobre la relación entre innovación e internacionalización (ej. Cassiman et al., 2010; Roper \& Love, 2002; Wakelin, 1998), vínculo que se comprueba también en este caso, lo que sugiere que debería estudiarse la mejor manera de articular las políticas de apoyo a una y otra actividad.

De cualquier manera, el análisis debe ser tomado con cuidado ya que presenta algunas limitaciones. Si bien la muestra de empresas de servicios tiene una amplia cobertura, no abarca a todos los subsectores. No se consideran empresas con menos de 4 años de existencia ni a aquellas empresas que dejaron de funcionar entre uno y otro período. Esto último podría estar introduciendo un sesgo al solo considerar las empresas "supervivientes".

Como sugerencias para futuras investigaciones, resulta interesante la utilización de otras técnicas econométricas que permitan avanzar en el análisis de causalidad, como son el análisis de panel de datos o el emparejamiento por puntaje de propensión (Aboal \& Garda, 2015). De la misma manera, es interesante profundizar en los resultados de la investigación sobre la interacción entre internacionalización e innovación, a los efectos de aportar a un mejor diseño y gestión de políticas.

Adicionalmente, es importante remarcar la importancia y necesidad de otro tipo de estudios para evaluar y comprender mejor el fenómeno 
de la innovación y el efecto de las políticas de apoyo. Si bien los análisis econométricos arrojan resultados relevantes, el seguimiento a empresas receptoras de apoyo podría dar la oportunidad de comprender mejor los factores de éxito, lo que conformaría un insumo valioso para el diseño y la gestión de estas políticas.

\section{Referencias}

Aboal, D., Angelelli, P., Crespi, G., López, A., Vairo, M., \& Pareschi, F. (2015). Innovación en Uruguay: diagnóstico y propuestas de política. Uruguay+25 Documento de trabajo No12.

Aboal, D., \& Garda, P. (2015). ¿La financiación pública estimula la innovación y la productividad? Una evaluación de impacto. Revista CEPAL 115 .

Aerts, K., \& Czarnitzki, D. (2004). Using Innovation Survey Data to Evaluate R\&D Policy: The Case of Belgium. SSRN Electronic Journal. https://doi.org/10.2139/ssrn.572086

Afcha, S. (2012). Analyzing the interaction between R\&D subsidies and firm's innovation strategy. Journal of Technology Management and Innovation. vol. 7(3). https://doi.org/10.4067/s071827242012000300006

Andrews, D., \& Criscuolo, C. (2013). Knowledge-Based Capital, Innovation and Resource Allocation. OECD Economics Department Working Papers No 1046. https://doi.org/10.1787/5k46bj546kzs-en

Bakhshi, H., Edwards, J., Roper, S., Scully, J., Shaw, D., Morley, L. \& Rathbone, N. (2013). Creative Credits - A Randomized Controlled Industrial Policy Experiment. London: Nesta.

Camacho, M., Jung, A., Horta, R., \& García, S. (2010). ¿Cómo innovan las empresas exitosas en Uruguay?: una aplicación del modelo 'la cometa de la innovación'. Montevideo, Universidad Católica del Uruguay (ISBN 978-9974-631-31-1).

Cassiman, B., Golovko, E., \& Martínez-Ros, E. (2010). Innovation, exports and productivity. International Journal of Industrial Organization, Elsevier, vol. 28(4), p.p. 372-376, July. https://doi.org/10.1016/j. ijindorg.2010.03.005

Cohen, W.M., \& Levinthal, D.A. (1989). Innovation and learning: The two faces of R\&D. Economic Journal, 99: 569-596. https://doi.org/10.2307/2233763

Cowling, M. (2016). You can lead a firm to R\&D but can you make it innovate? UK evidence from SMEs. Springer Science+Business Media, New York. https://doi.org/10.1007/s11187-016-9704-2

Cunningham, P., Edler, J., Flanagan K., \& Laredo, P. (2013). Innovation policy mix and instrument interaction: a review. Nesta Working Paper No. 13/20.
Czarnitzki, D. \& Lopes-Bento, C. (2014). Innovation Subsidies: Does the Funding Source Matter for Innovation Intensity and Performance? Empirical Evidence from Germany. Industry and Innovation, Taylor \& Francis Journals, vol. 21(5), 380-409, July. https://doi.org/10.2139/ssrn.1908764

Czarnitzki, D., Hanel, P., \& Rosa, J.M. (2011). Evaluating the Impact of R\&D Tax Credits on Innovation: a Microeconometric Study on Canadian Firms. Research Policy, vol. 40, N² , Amsterdam, Elsevier. https://doi.org/10.2139/ssrn.651341

Czarnitzki, D., \& Hussinger, K. (2004). The Link Between R\&D Subsidies, R\&D Spending and Technological Performance. ZEW Discussion Paper, $\mathrm{N}^{\circ}$ 04-056, Mannheim, Centro de Investigación Económica Europea. https://doi.org/10.2139/ssrn.575362

David, P., Hall, B.H., \& Toole, A.A. (2000). Is Public R\&D a Complement or Substitute for Private R\&D? A Review of the Econometric Evidence, Research Policy, vol. 29, N 4-5, Amsterdam, Elsevier. https://doi.org/10.3386/w7373

Dogson, M., Hughes, A., Foster, J., \& Metcalfe, S. (2011). Systems thinking, market failure, and the development of innovation policy: The case of Australia. Research Policy, 40 1145-1146. https://doi.org/10.1016/j.respol.2011.05.015

Durán, C, Camacho, M., Jung, A., \& Karsaclian, D. (2016). Aportes para una discusión de políticas de fortalecimiento de PYMEs innovadoras y dinámicas en América Latina”, Programa de Políticas Sociales y Económicas para América Latina, Konrad-Adenauer-Stiftung (de próxima publicación).

Edler, J., Cunningham, P., Gök, A., \& Shapira, P. (2013). Impacts of Innovation Policy: Synthesis and Conclusion. Nesta Working Paper No. 13/21.

Edquist, C. (2011). Design of innovation policy through diagnostic analysis: identification of systemic problems (or failures). Industrial and Corporate Change, 20(6), 1725-1753. https://doi.org/10.1093/icc/dtr060

Flanagan, K., Uyarra, E., \& Laranja, M. (2011). Reconceptualising the 'policy mix' for innovation. Research Policy, 40 702-713. https://doi.org/10.1016/j.respol.2011.02.005

Freeman, C. (1987). Technology and Economic Performance: Lessons from Japan. Pinter Publishers, London.

García-Granero, A., Vega-Jurado, J., \& Alegre-Vidal, J. (2014). Is R\&D enough to take advantage from external Knowledge? Focusing on coordination mechanisms. Journal of Technology Management \& Innovation, vol. 9(2). https://doi.org/10.4067/s0718-27242014000200009

González-Pernía, J.L., Jung, A., \& Peña, I. (2015). Innovation-driven entrepreneurship in developing economies. Entrepreneurship and Regional Development, vol. 27(9-10), pages 555-573, October. https://doi.org/10.1080/08985626.2015.1075602 
Hall, B.H., \& Lerner, J. (2009). The Financing of R\&D and Innovation. NBER Working Paper No. 15325. https://doi.org/10.3386/w15325

Hall, B.H., \& Maffioli, A. (2008). Evaluating the Impact of Technology Development Funds in Emerging Economies: Evidence from Latin America, OVE Working Papers, $N^{\circ}$ 0108, Washington, D.C., IDB. https://doi.org/10.3386/w13835

Hausmann, R., \& Klinger, B. (2006). Structural Transformation and Patterns of Comparative Advantage in the Product Space. SSRN Electronic Journal. https://doi.org/10.2139/ssrn.939646

Hausmann, R., \& Rodrik, D. (2003). Economic development as selfdiscovery. Journal of Development Economics, vol. 72, No. 2, Amsterdam, Elsevier, December. https://doi.org/10.3386/w8952

Imbs, J., \& Wacziarg, R. (2003). Stages of Diversification. American Economic Review, 93(1): 63-86. https://doi.org/10.2139/ssrn.251948

Magro, E., \& Wilson, J.R. (2013). Complex innovation policy systems: Towards an evaluation mix. Research Policy, 42, 1647-1656. https:// doi.org/10.1016/j.respol.2013.06.005

Maloney, W.F. (2002). Missed opportunities: innovation and resource based growth in Latin America. Economia, vol. 3, No. 1, Washington, D.C., Brookings Institution Press.

Maloney, W.F., \& Perry, G. (2005). Towards an efficient innovation policy in Latin America. CEPAL Review n ${ }^{\circ}$ 87, p. 25-43.
Metcalfe, J. (1995a). Technology Systems and Technology Policy in an Evolutionary Framework. Cambridge Journal of Economics, 19(1): 25-46. https://doi.org/10.1093/oxfordjournals.cje.a035307

Navarro, J.C., Benavente, J.M., \& Crespi, G. (2016). The New Imperative of Innovation: Policy Perspectives for Latin America and the Caribbean. IDB. https://doi.org/10.18235/0000245

OCDE (2010). The Innovation Policy Mix. En OCDE, OECD Science, Technology and Industry Outlook 2010 (pp. 251-279). OECD, Paris.

Padilla-Pérez, R., \& Gaudin, Y. (2014). Science, Technology and Innovation Policies in Small and Developing Economies: The Case of Central America. Research Policy 43: 749-59. https://doi.org/10.1016/j.respol.2013.10.011

Roper, S., \& Love, J.H. (2002). Innovation and export performance: evidence from the UK and German manufacturing plants. Research Policy, Elsevier, vol. 31(7), p.p 1087-1102, September. https://doi.org/10.1016/s0048-7333(01)00175-5

Szczygielski, K., Grabowski, W., Pamukcu, M.T., \& Tandogan, V.S.. (2017). Does government support for private innovation matter? Firm level evidence from two catching-up countries. Research Policy, 46: 219-237. https://doi.org/10.1016/j.respol.2016.10.009

Wakelin, K. (1998). The role of innovation in bilateral OECD trade performance. Applied Economics, Taylor \& Francis Journals, vol. 30(10), pages 1335-1346. https://doi.org/10.1080/000368498324959 